\title{
Investigation of Energy Losses in the Flow Section of the Small-Sized Axial-Flow Pump
}

\author{
Alexander Gusak ${ }^{1,}$, Olga Matvienko ${ }^{1, b^{*}}$, Victor Nenja ${ }^{1, c}$ \\ ${ }^{1}$ Department of Applied Fluid Mechanics, Sumy State University, Rimskogo-Korsakova str., 2, \\ Sumy, Ukraine, 40007 \\ agusak@pgm.sumdu.edu.ua, ${ }^{b}$ matvienko@pgm.sumdu.edu.ua, ${ }^{c}$ nenja_vg@mail.ru
}

Keywords: energy losses, submersible pump, small-sized axial stage, numerical simulation, efficiency.

\begin{abstract}
The paper present results of a study of a small-sized axial-flow stage with blades of small aspect ratio, which is designed for submersible borehole pumps. The energy losses in the flow section of stage are analyzed in research. Refined equations of total hydraulic losses for these flow parts were received by numerical simulation. The results of numerical simulation are compared with experimental data.
\end{abstract}

\section{Introduction}

Problems of population provision with qualitative drinking water occurred and were solved during lots of years of Ukraine water supply system being, that is why problem of maximal effective equipment for water production, able to work with minimal capital costs and minimal energy resources cost, is still actual. Solution of this problem has been never closed and scientific-research progress facilitates appearance of more modern mechanisms, development of new technologies and water preparation methods.

One of such modern solutions is application of low specific speed stages in borehole pumps; they have a range of advantages (high hydraulic efficiency, simple and compact construction, raised resource of support node and pump in whole). But determinant advantage of axial working elements application in borehole pumps is that they afford possibility for invariable diametric sizes to increase feed in several times comparing with existing constructions, which allows decrease of borehole cost in several times for the same volume of produced water.

Obstacle for wide application of such stages is absence of experience of their development and well-tested range of such type flow parts for different parameters.

Single-stage constructions of axial pumps are well-known and widely used in practice. They have simple construction, low mass and compactness, providing high feed.

Multi-stage axial pumps are used in rocket-building, namely in aggregates for fluid rocket engines power [1]. High circular velocities, high values of specific work, aggressive working environment and raised requirements to cavitation stability are typical for these pumps.

Efforts of multi-stage axial pumps application in oil-gas and mining industry for multi-phase liquids pumping are well-known too $[2,3]$.

Advantages of axial stages with low specific speed for borehole pumps were studied in the papers [4-6], methods of their designing were developed, analysis of numerical modeling results and full-scale tests of specimen was implemented and this afforded an opportunity to ground relevance of such type stages application for artesian water supply systems.

\section{Main part}

It is necessary to consider workflow flow parts for development of complex method for smallsized axial stages with low specific speed designing with small lengthening blades. 
Workflow of pump intermediate stage flow part represents transformation and redistribution of each form of energy that is why model of energy distribution can be applied as its model.

Considering that reactivity coefficient of analyzed impeller equals 0,85 (it means that $85 \%$ of specific fluid energy transforms into potential pressure energy, naturally $15 \%$ of energy transforms in stator in this impeller), it can be said that basic hydraulic losses will take place namely in the impeller. Consequently principal attention is paid to small-sized axial impeller in this paper.

We will use the following equation for definition of head of the stage:

$$
H_{c m}=H_{T R K}-S Q^{2} \text {, }
$$

where $H_{T R K}$ - theoretical head of the impeller;

$Q$ - flow rate;

$S=f\left(\zeta_{\Sigma}\right)$ - coefficient, which includes head losses, where $\zeta_{\Sigma}$ - total losses coefficient.

Hydraulic losses are naturally complete, that is why they are complex from the point of view of their theoretical and experimental research. Complicated physical processes, which take part in dynamic pumps, are the main reason of it. Flow of viscous fluid generally is accompanied by energy dispersion that is hydraulic losses. Viscous fluid resistance to flow part channels modifications is the reason of these losses occurrence.

In general, hydraulic losses in flow part of small-sized axial stage can be presented:

$$
\sum \Delta h=\Delta h_{R K}+\Delta h_{S A} \text {, }
$$

where $\Delta h_{R K}$ - head losses in the impeller;

$$
\Delta h_{S A}-\text { head losses in stator. }
$$

As $\left.\left.\Delta h_{R K}\right\rangle\right\rangle \Delta h_{S A}$, so $\sum \Delta h=\sum \Delta h_{R K}=\zeta_{R K} \frac{V_{m}^{2}}{2 g}[7,8]$.

There are several types of losses in inter-blade channel of axial stage: profile, front, secondary and slot. Every type of losses is characterized by appropriate loss factor. Mentioned above loss coefficients are often defined by the known formulas [9].

Although, according to the investigations [10], given dependences not always provide accurate results, especially for impellers with small lengthening blades. Taking into consideration that investigated flow part has a range of features, it is necessary to specify formulas for head losses coefficients definition.

Any blading machine, including pump with small-sized axial stages of low specific speed, can be regarded as combination of consistently joint immovable channels and rotating channels, profiles of different form and length, and each of them has specified hydraulic losses.

To calculate losses, scheme of three components division is usually applied in such cases, the components are: head losses, generated by channel walls friction $\Delta h_{f}$, losses, relating to channel expansion $\Delta h_{c}$, and losses, relating to flow turne. The last component is not considered, because it is identical for all values of $h^{\prime}$ for fixed value of sleeve ratio. Besides, losses in radial clearance should be considered. It is considered that flow leakage into blades occurs with zero angle of attack.

Concept of equivalent diffuser, in which axial length, squares of inlet and outlet intersections are equal to corresponding parameters of outlet diffuser channel, is often used for approximate estimation of hydraulic resistance of diffuser channels of complicated form [9, 10]. Application of two geometrical parameters of equivalent diffuser, namely opening angle and expansion ratio, is widely used in engineering calculations. Although this method doesn't provide accurate results for flow parts, formed by small lengthening blades (small-sized axial stages with low specific speed). Concept of local angle of diffuser opening was introduced in the papers [9, 10], which provides opportunity to increase accuracy of obtained results. This approach is used for estimation of stage operation effectiveness.

The following formula of local angle of equivalent inter-stage channel diffuser opening was obtained in the paper [11]: 


$$
\alpha_{m}=\operatorname{arctg}\left(\frac{h^{\prime} \sin \left(\beta_{l 1}-\frac{l}{R}\right) \sin \left(\frac{\Theta}{2}\right)}{h^{\prime}\left(\frac{b}{t}\right)+\cos \left(\beta_{l 1}-\frac{l}{R}\right)}\right),
$$

where $R$ - radius of blade arch; $l$ - length; $\beta_{l 1}$ - angle of blade arrangement; $\Theta$ - angle of blade girth; $b / t$ - cascade density.

Dependences of local angle of diffuser opening from specific length of impeller channel of small-sized axial stage with low specific speed are given in the figure 1. Specific length of channel is relation of channel longitudinal coordinate to maximal channel length $\left(l^{\prime}=\frac{l}{l_{\max }}\right.$, where $l-$ channel longitudinal coordinate, $l_{\max }$ - maximal channel length).

Calculations of angle $\alpha_{\mathrm{M}}$ for investigated impellers show that $\alpha_{\mathrm{M}}$ changes along channel axe according to linear law, decreasing to channel exit; gradient of this value is gradually growing with increase of specific blade height. It should be mentioned that character of given curves is lowvariable when profile solidity is taken into account (for small specific thickness of profile).

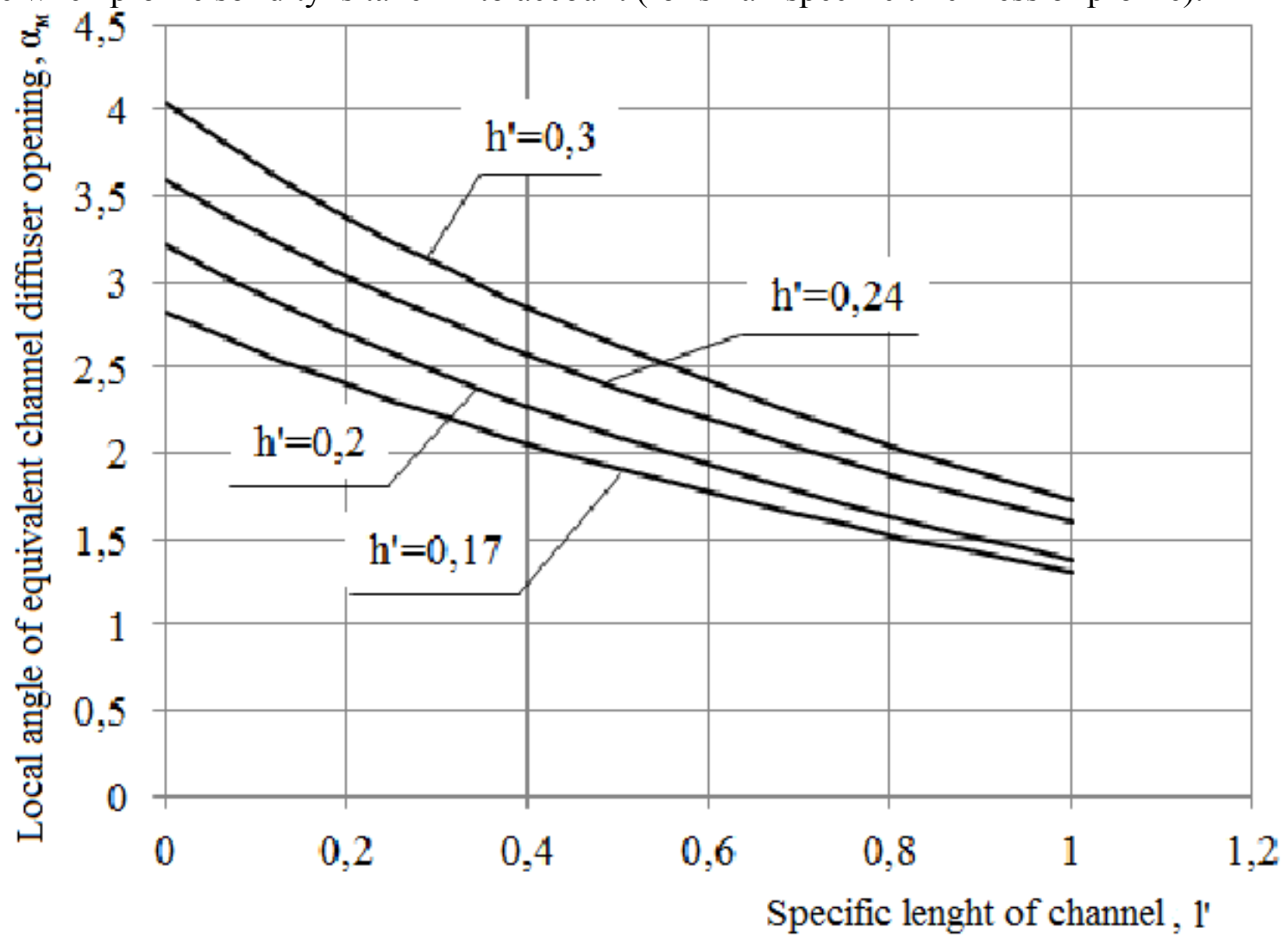

Fig. 1. Dependence of local angle of diffuser opening from specific length of channel

Qualitative estimation of head losses for different specific heights of blades were implemented using formula for $\alpha_{\mathrm{M}}$, like it is implemented for curvilinear diffusers consideration.

Losses coefficients relating to friction and flow rotation are defined by local angles of equivalent diffuser opening along axial line of inter-stage channel, considering that parameters of cascade and flow at the mean radius are typical for the whole blade. It was taken into consideration, that losses coefficient relating to expansion doesn't depend on angles of attack of cascade profiles.

Losses coefficients in inter-stage channels of investigated impellers for angle of attack $\alpha=0$ were defined according to the formulas [9]. Data of corresponding calculations is given in the figure 2. 


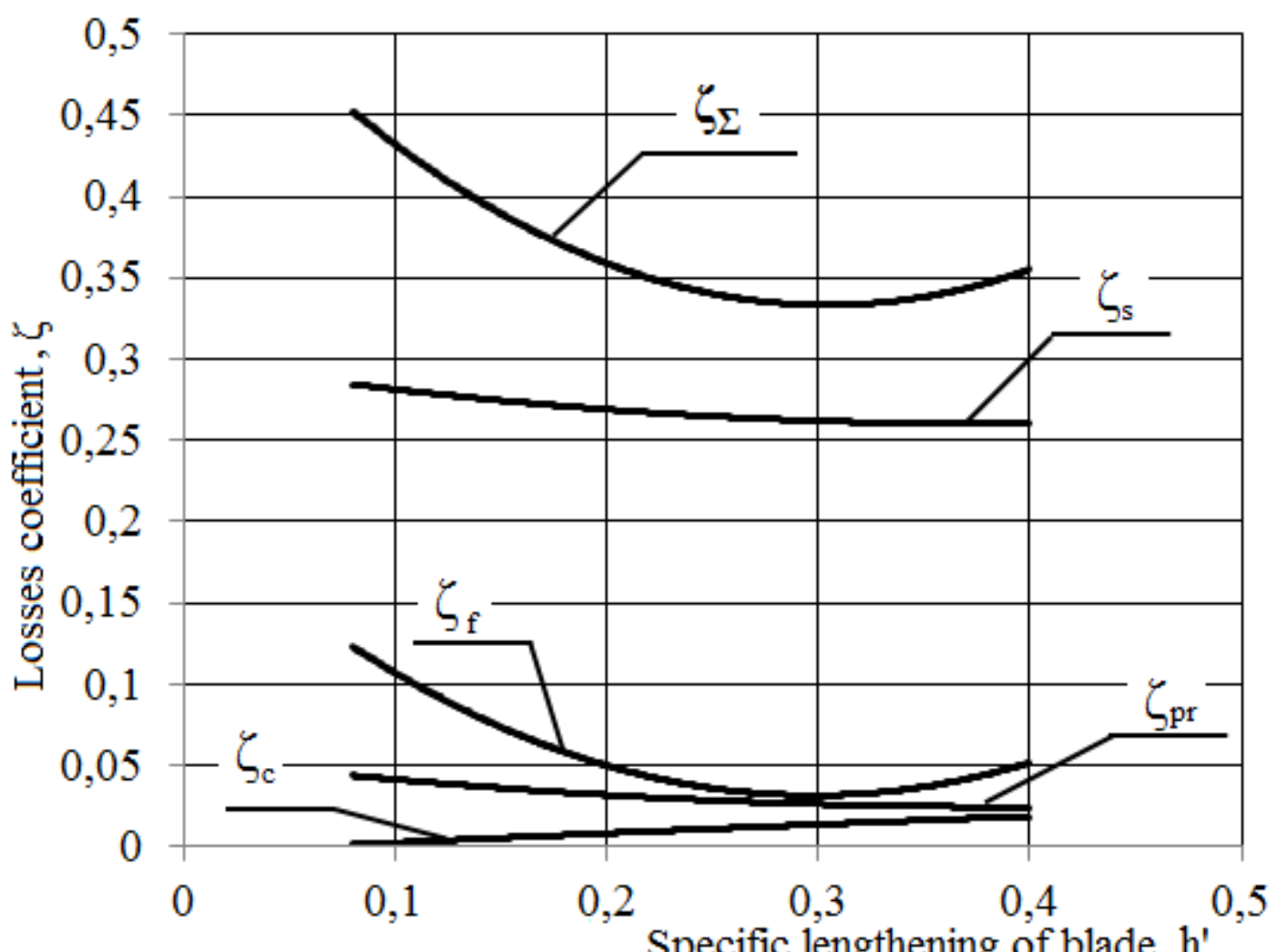

Fig. 2. Dependence of losses coefficient in inter-stage channel of small-sized axial impeller with low specific speed from specific lengthening of blade (where $\zeta_{p r}$-profile losses coefficient; $\zeta_{f}$ - friction losses coefficient; $\zeta_{c}-$ channel expansion losses coefficient $\zeta_{s}-$ slot losses coefficient; $\zeta_{\Sigma}-$ total losses coefficient)

Analyzing curves, given in the figure 2, it should be mentioned, that obtained curve of total losses coefficient has clearly defined minimum, which spans in the range of specific lengthening of blade values $0,2-0,4$. Besides, head losses coefficient in radial clearance has maximal values of all four components of total losses coefficient.

\section{Analysis of the work results}

Implemented calculations of local angle of diffuser denote that decrease of specific height of blade leads to decrease of total diffuser ability of channel. Besides, one of methods of row effectiveness increase is profiling, which provides minimal values of $\alpha_{M}$ and its gradient in any point.

Approximating curves, given on the figure 2 by means of computer with mean square error less than $1 \%$, the following system of equations was obtained:

$$
\left\{\begin{array}{l}
\varsigma_{f}=2\left(h^{\prime}\right)^{2}-1,15 h^{\prime}+0,2 \\
\varsigma_{s}=0,27\left(h^{\prime}\right)^{2}-0,2 h^{\prime}+0,3 \\
\varsigma_{p r}=0,2\left(h^{\prime}\right)^{2}-0,15 h^{\prime}+0,06 \\
\varsigma_{c}=-0,02\left(h^{\prime}\right)^{2}-0,06 h^{\prime} \\
\varsigma_{\Sigma}=2,45\left(h^{\prime}\right)^{2}-1,56 h^{\prime}+0,56
\end{array}\right.
$$

Thereby, it was defined that small-sized axial stages with low specific speed should be designed with specific lengthening of blade in the range of 0,2-0,4. Hydraulic losses in this range are minimal.

From formula (4) head losses in impeller of investigated stage can be obtained: 


$$
\Delta h=\zeta_{\Sigma} \frac{V_{m}^{2}}{2 g}
$$

where $\zeta_{\Sigma}-$ is calculated according to formula (4).

Preliminary check and confirmation of relation (5) accuracy was implemented based on the results of numerical simulation (by means of software ANSYS CFX) of mathematical model of pump workflow, and its adequacy was proved by results of experimental research [10].

Typical result of comparison of rated values of head losses in small-sized axial impeller with low specific speed and corresponding values, obtained by means of numerical experiment depending on specific axial component of absolute velocity, is given in the figure 3.

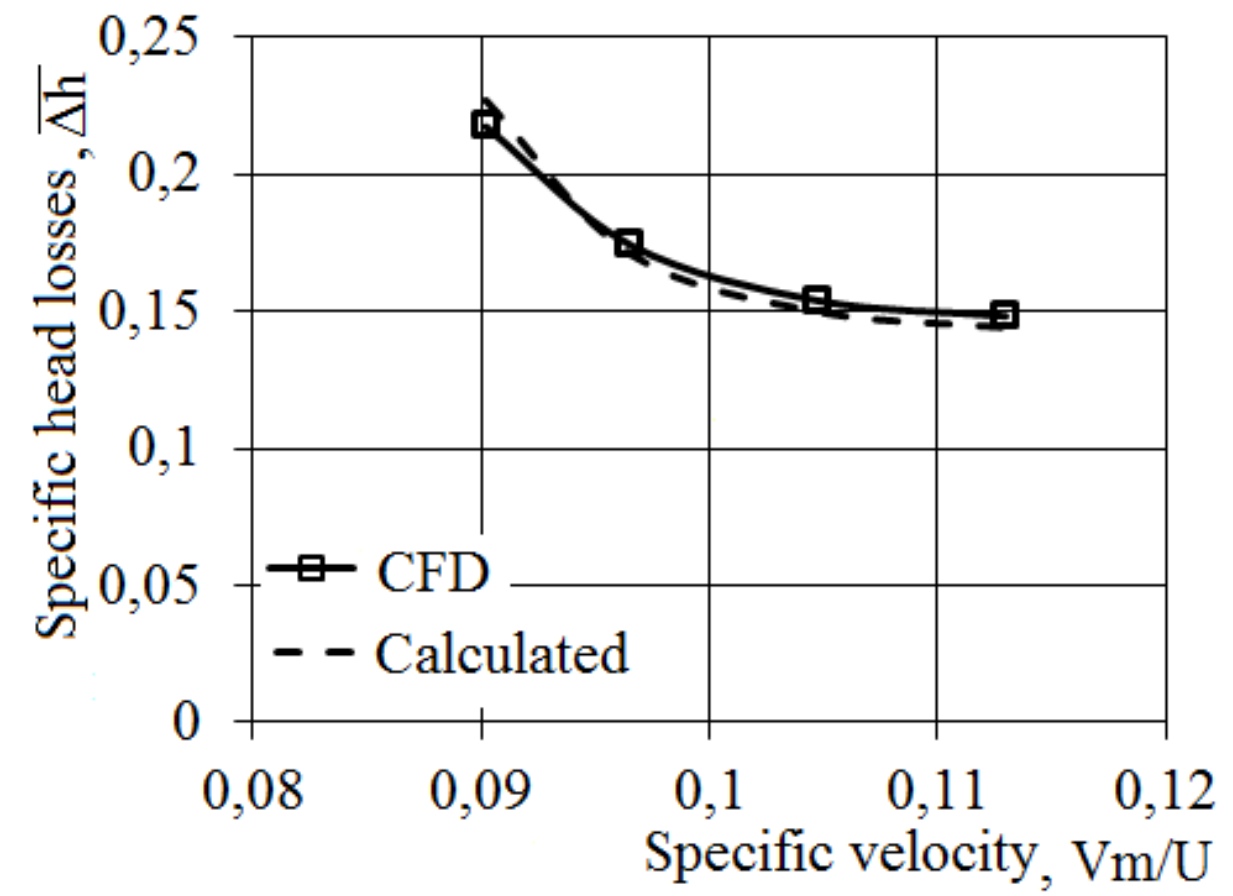

Fig. 3. Typical result of comparison of values of specific head losses $\left(\overline{\Delta h}=\frac{\Delta h}{H_{r}}\right.$, where $\Delta \mathrm{h}-$ head losses value, $\mathrm{H}_{\mathrm{r}}$ - rated value of stage head), calculated according to the formulas (4), (5) and obtained by numerical simulation

Analyzing obtained diagram, it is obvious that divergence of obtained results is less than $7 \%$, that is sufficient to apply formulas (4) and (5) for definition of head losses in investigated flow parts. Thereby, refined mathematical model of head losses distribution in flow part of small-sized axial stage with low specific speed can be considered as valid. Final confirmation was implemented by means of physical experiment [10].

\section{Conclusions}

Mathematical model of workflow of small-sized axial stage with low specific speed with small lengthening blades was refined in this paper. Structure of hydraulic losses in channel of investigated stage was defined according to the results of analytical research and numerical simulation of flow in flow part. Analytical dependences were obtained for head losses coefficients. Rational range of specific lengthening of blade of investigated impellers was defined $\left(h^{\prime}=0,2-0,4\right)$. 


\section{References}

[1] K. Kamijo, N. Sakazume, Sh. Warashina, Developmental History of Liquid Oxygen Turbopumps for the LE-7 Engine, Trans. Japan Soc. Aero. Space Sci., Vol. 44, No. 145 (2001) $155-163$.

[2] F. Fedcov, New multiphase pumps for reservoir pressure maintenance systems with gas content of up to 95\%, Theory and Practice of pump and compressor design, Sumy, (2011) 293-299.

[3] A. Elin, Screw multistage pumps: design procedure, quality indicators, Doctor of Philosophy Dissertation, Sumy, Ukraine, 2002.

[4] A. Gusak, O. Demchenko, I.Kaplun, Prospects of use axial pumps for artesian water, Industrial hydraulics and pneumatics, Vol. 4, 30 (2010) 8-11.

[5] O. Demchenko, A. Gusak, I. Kaplun, Investigation of Small-Sized Axial-Flow Stage of a Borehole Pump for Water Supply, Proceedings of the 4th International Meeting on Cavitation and Dynamic Problems in Hydraulic Machinery and Systems, (2011) 143-150.

[6] I. Kaplun, S. Lugova, O. Matvienko, Creating a dimension-series of new type submersible borehole pumps, Scientific Bulletin of the building, Vol.70, (2012) 326-333.

[7] A. Gusak, Improving the flow parts of monoblock submersible pumps with high specific speed, Doctor of Philosophy Dissertation, Sumy, Ukraine, 1996.

[8] S. Dovshcik, Research of low-speed axial blowers, Industrial aerodynamics, Mashinostroenie, Moscow, (1991) 5-29.

[9] A. Gegin, Effect of aspect ratio of the blades on the properties of the axial compressor, Works of TsAGI, Vol. 29 (1973) 35-55.

[10] O. Matvienko, Small-sized axial-flow stage of the borehole pump with increased energy efficiency, Doctor of Philosophy Dissertation, Sumy, Ukraine, 2013. 